Portland State University

PDXScholar

\title{
Automated Nanocrystal Orientation and Phase Mapping in the Transmission Electron Microscope on the Basis of Precession Electron Diffraction
}

\author{
Edgar F. Rauch \\ SIMAP/GPM2 Laboratory \\ Joaquin Portillo \\ NanoMEGAS SPRL \\ Stavros Nicolopoulos \\ NanOMEGAS SPRL \\ Daniel Bultreys \\ NanoMEGAS SPRL \\ Sergei Rouvimov \\ SERVEIS Cientificotecnics \\ Follow this and additional works at: https://pdxscholar.library.pdx.edu/phy_fac

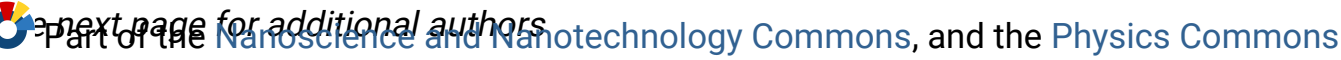 \\ Let us know how access to this document benefits you.
}

\section{Citation Details}

Rauch, Edgar F., et al. "Automated nanocrystal orientation and phase mapping in the transmission electron microscope on the basis of precession electron diffraction." Zeitschrift für Kristallographie International journal for structural, physical, and chemical aspects of crystalline materials 225.2-3 (2010): 103-109.

This Article is brought to you for free and open access. It has been accepted for inclusion in Physics Faculty Publications and Presentations by an authorized administrator of PDXScholar. Please contact us if we can make this document more accessible: pdxscholar@pdx.edu. 


\section{Authors}

Edgar F. Rauch, Joaquin Portillo, Stavros Nicolopoulos, Daniel Bultreys, Sergei Rouvimov, and Peter Moeck 


\title{
Automated nanocrystal orientation and phase mapping in the transmission electron microscope on the basis of precession electron diffraction
}

\author{
Edgar F. Rauch*, I, Joaquin Portillo ${ }^{\text {II, III }}$, Stavros Nicolopoulos ${ }^{\text {II }}$, Daniel Bultreys ${ }^{\text {II }}$, Sergei Rouvimov ${ }^{\text {III }}$ and Peter Moeck ${ }^{\text {IV }}$ \\ I SIMAP/GPM2 laboratory, CNRS-Grenoble INP, BP 46101 rue de la Physique, 38402 Saint Martin d'Hères, France \\ II NanoMEGAS SPRL, Boulevard Edmond Machterns No 79, Saint Jean Molenbeek, Brussels, 1080, Belgium \\ III SERVEIS Cientificotecnics, University of Barcelona, Spain \\ IV Nano-Crystallography Group, Department of Physics, Portland State University, \\ and Oregon Nanoscience and Microtechnologies Institute Portland, OR 97207-0751, USA
}

Received July 4, 2009; accepted November 13, 2009

\section{Nanocrystals / Orientation mapping / Structural mapping / Structural fingerprinting / Structural databases / \\ Precession electron diffraction / \\ Transmission electron microscopy}

\begin{abstract}
An automated technique for the mapping of nanocrystal phases and orientations in a transmission electron microscope is described. It is primarily based on the projected reciprocal lattice geometry that is extracted from electron diffraction spot patterns. Precession electron diffraction patterns are especially useful for this purpose. The required hardware allows for a scanning-precession movement of the primary electron beam on the crystalline sample and can be interfaced to any older or newer mid-voltage transmission electron microscope (TEM). Experimentally obtained crystal phase and orientation maps are shown for a variety of samples. Comprehensive commercial and open-access crystallographic databases may be used in support of the nanocrystal phase identification process and are briefly mentioned.
\end{abstract}

\section{Introduction}

The electron backscatter diffraction (EBSD) attachment for scanning electron microscopes (SEM) has gained popularity over the last decade as it provides an effective tool for the characterization of bulk crystalline materials. EBSD crystal phase and orientation maps with a resolution of typically $50 \mathrm{~nm}$ when a field emission gun is used have been recorded mainly from metals and alloys. The technique is based on precise measurements of backscattered Kikuchi line patterns. The quality of these patterns is known to be sensitive to the surface roughness so that efficient mapping requires well polished samples. Another limitation of the Kikuchi lines is related to their disappearance with increasing strain and internal stresses.

* Correspondence author (e-mail: edgar.rauch@simap.grenoble-inp.fr)
On the other hand, there is a growing need to characterize, map and fingerprint nanocrystalline materials at higher resolutions as obtainable in a transmission electron microscope (TEM). Consequently, the extension of orientation mapping facilities to transmission electron microscopes (TEM) was attempted several times. Both Kikuchi lines [1-3] and Bragg diffraction (spot) patterns [2, 4] were used to extract the orientation and phase information from the patterns. Such techniques have become more feasible due to the availability of focused ion beam (FIB) microscopes for the preparation of sufficiently thin foils. Nevertheless, these techniques suffer from difficulties to analyze diffraction patterns that are sensitive to dynamical effects related for example to crystal thickness changes. Moreover, the complexity of the patterns that are collected when the electron beam is crossing overlapping grains leads to frequent mis-indexings.

There is an alternative TEM technique that works on the basis of approximately 65,000 reconstructed diffraction spot patterns per mapped sample area $[5,6]$. These patterns are reconstructed by software from some 5,000 automatically collected conical dark field images. Only the projected reciprocal lattice geometry of the reconstructed diffraction patterns is utilized. The technique is commonly referred to as "Automated Crystallography for the TEM" [7]. Further improvements of this technique that include utilizing the intensity of the reconstructed diffraction patters in the two-beam dynamical diffraction limit and a Blackman correction have been proposed recently [8].

It was proposed previously to use a template matching algorithm to compare the acquired diffraction patterns to pre-calculated templates [9]. This permits the crystal orientation and phase to be derived form the best fit among a complete set of possibilities. Such an approach has proved able of deconvoluting superimposed diffraction patterns that were recorded from grain or twin boundaries [10]. The efficiency of template matching for orientation indexing was recognized by other groups that are dealing either with electron [8] or Laue X-ray [11] diffraction patterns. 

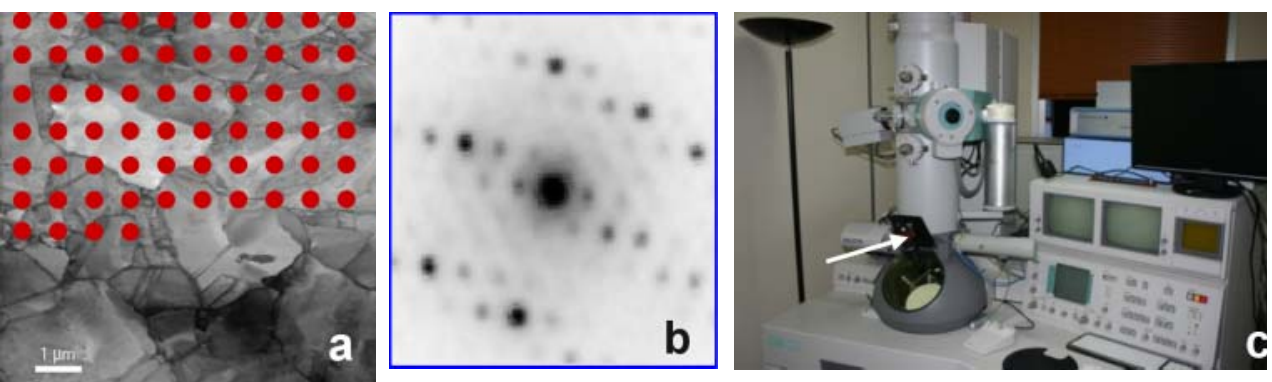

Fig. 1. Automatic TEM nanocrystal phase and orientation mapping. While the area of interest is scanned and precessed (a), ED spot patterns are collected (b) with an external CCD camera that is mounted in front of the TEM screen (arrow in c). The scanning of the primary electron beam is combined with its precession movements, both of which are controlled by the dedicated digital "DigiSTAR" unit (d). The array of spots in (a) are supposed to illustrate the scanning procedure (Note that this array is not to scale as typical scan step sizes are in the tens of nanometer).
In this paper, we will describe both this method and a newly developed tool for the automatic mapping of crystals orientations and phases [9, 10, 12]. Our method may be loosely speaking referred to as a "higher resolution EBSD-TEM equivalent" or better as precession-transmission-diffraction based "Orientation Imaging Microscopy".

While template matching is a relatively simple and fast procedure, it cannot deal with the so-called $180^{\circ}$ ambiguity that pertains to some particular zone axes. The problem results from the absence of reflections pertaining to higher order Laue zone in typical diffraction spot patterns. The missing reflections may be activated when the incident beam is tilted. Consequently, precessing the transmitted beam with an angle of a few tens of a degree up to a few degrees is expected to decrease the ambiguity. The benefits of such precession movements of the primary electron beam on the sample and a proper de-scanning below the sample on the reliability of the orientation measurement will be demonstrated.

\section{Materials and methods}

Structural information about crystalline materials is contained in their electron diffraction (ED) patterns. Kikuchi lines and/or individual diffraction spots may be present in such patterns. For our TEM-based technique [9, 10, 12] we are utilizing ED spot patterns exclusively. Crystal orientation and phase determinations from ED spots will be less accurate that from Kikuchi lines, but this disadvantage (with respect to EBSD-SEM) is compensated by the method inherent insensitivity to local strains and rough surfaces that frequently impede EBSD-SEM analyses.

The ED spot patterns are collected sequentially with a charged coupled device (CCD) camera, Fig. 1, while the sample area (that is typically tens of square micrometers) is scanned by the primary electron beam and simultaneously precessed around the optical axis of the micro- scope. The beam scanning and precessing is controlled by a dedicated external device, i.e. the precession electron diffraction device "DigiSTAR" [13], via adequate connections to the deflector coils control boards. A TEM retrofitted with a precession device, thus, does not need to possess scanning facilities itself.

Any slow-scan CCD camera that is mounted on an external $35 \mathrm{~mm}$ standard port or on-axis internally can be used for the collection of precession ED spot patterns with a speed of about 10 to 30 frames per seconds. An external "fast optical" CCD with only 8 bit dynamical range and $750 \times 500$ pixels image size that is mounted in front of the TEM screen can be used advantageously because of its much higher collection speed. Using the camera binning facility, up to $180 \mathrm{ED}$ spot patterns can be recorded per second with the current equipment. During the scanning and precessing of the primary electron beam, thousands of ED spot patterns are recorded and stored in a computer's memory.

In order to proceed with nanocrystal orientation and phase identification (or utilize it for more advanced ${ }^{l}$ struc-

1 A novel procedure for structural fingerprinting of nanocrystals on the basis of structural data that can be extracted from precession electron diffraction spot patterns is proposed in Refs. [14-16]. This procedure possesses three hierarchical levels: extraction and utilization of (i) the projected reciprocal lattice geometry, (ii) the projected point symmetry, and (iii) estimates of the structure factor moduli. All three levels benefit from a precessing primary electron beam. Crystalline materials that have very similar projected reciprocal lattice geometries may well differ in their projected point symmetries and structure factor moduli (even if they have the same element content and a similar stoichiometry). A mixture of nanocrystalline magnetite $\left(\mathrm{Fe}_{3} \mathrm{O}_{4}\right)$ and maghemite $\left(\gamma-\mathrm{Fe}_{2} \mathrm{O}_{3}\right)$ can serve here as an example (Moeck, P.; Rouvimov, S.; Nicolopoulos, S.; Oleynikov, P.: Structural fingerprinting of a cubic iron-oxide nanocrystal mixture: A case study. NSTI-Nanotech 2008, Vol. 1, (2008) 912-915 (www.nsti.org, ISBN 978-1-4200-8503-7); open access version of a similar conference paper: arXiv:0804.0063). Individual members of both nanocrystalline phases could be distinguished, i.e. fingerprinted structurally in other words, on the basis of high resolution phase contrast transmis- 

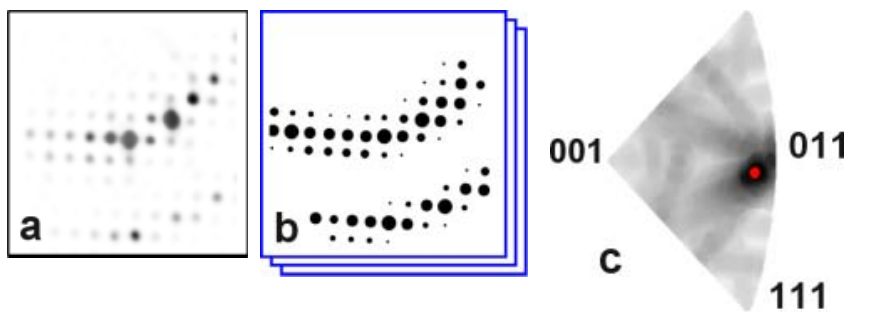

Fig. 2. Indexing is performed by cross-correlating (a) the experimentally acquired ED spot pattern with (b) the simulated templates for all possible orientation and crystal phases. The degree of matching between an experimental ED pattern and a given simulated template is given by the correlation index. The highest value determines the adequate crystal orientation and phase. The Q-map, here shown for a crystal whose point group is $\overline{4} 3 m(\mathbf{c})$, displays the relative values of the correlation indexes for all possible orientation as a gray level.

tural fingerprinting on the basis of precession electron diffraction data [14-16]) for each experimental ED spot pattern, thousands of simulated ED spot patterns (so called templates) are utilized for each crystallographic phase in the sample. The degree of matching for a given template is characterized by a correlation index that is defined by the following relation:

$$
Q(i)=\frac{\sum_{j=1}^{m} P\left(x_{j}, y_{j}\right) T_{i}\left(x_{j}, y_{j}\right)}{\sqrt{\sum_{j=1}^{m} P^{2}\left(x_{j}, y_{j}\right)} \sqrt{\sum_{j=1}^{m} T_{i}^{2}\left(x_{j}, y_{j}\right)}}
$$

where the highest value corresponds to the most probable orientation and phase, see also Fig. 2.

Here the experimental ED spot pattern is represented by the intensity function $P(x, y)$ while the template $i$ is given by the function $T_{i}(x, y)$. The $Q(i)$ values for a given pattern may be displayed in a so-called correlation index map ( $Q$ map) that is the portion of the stereographic projection that covers all the possible orientations for the given crystal symmetry. It turns out that for highly symmetric cubic structures (i.e. point groups $m \overline{3} m$, and $\overline{4} 3 m$ ) and for successive orientation templates differing by one degree, less than 1500 templates have to be generated to cover all of the orientation possibilities. In Fig. 2c, the local correlation indexes, normalized by the maximal encountered correlation index, are displayed in a gray scale where the darkest point in the map corresponds to the selected orientation.

The template matching procedure can also be used to select adequate indexing parameters when ED spot patterns with not precisely known observation conditions are analyzed. The software then automatically detects the best fit camera length from the experimental ED spot patterns.

sion electron microscopy images (Moeck, P.; Bjorge, R.; Mandell, E.; Fraundorf, P.: Lattice-fringe fingerprinting of an iron-oxide nanocrystal supported by an open-access database. Proc. NSTI-Nanotech Vol. 4 (2007) 93-96, ISBN 1-4200637-6-6). These images needed to be recorded from individual nanocrystals that were in a thickness range were the weak phase object approximation is applicable. Precession electron diffraction should allow for similar distinctions for such iron-oxide nanocrystals that are five to ten times thicker.
The software includes modules for (i) acquiring ED spot patterns, (ii) generating simulated templates, (iii) comparing templates by cross correlation (iv) indexing of acquired ED spot patterns, and (v) generating crystal phase and orientation maps. All maps and ED spot patterns are stored and can be revisited for further processing off-line.

For a typical map of $400 \times 400$ pixels, the beam scanning (and precessing) over the sample area may take only 25 minutes. The comparison with simulated templates can be done off-line and will takes about 20 minutes for highly symmetric cubic materials and 2 to 24 times longer for unit cells with lower symmetry because more templates must be generated and compared with experimental ED spot patterns for the same angular resolution. The spatial resolution may be as good as about half the primary electron beam size, e.g. $15 \mathrm{~nm}$ for a $30 \mathrm{~nm}$ spot size of a TEM with $\mathrm{LaB}_{6}$ electron gun.

The template generation is done on the basis of the crystal structure parameters of the known phases (for with the spatial distribution is to be mapped). The dimensions of the unit cell, the space group symmetry, and fractional atomic positions can be read from files in the standard Crystallographic Information File format [17, 18] (CIF, file extension *.cif). Both well-known commercial databases (also utilizing their free demonstration versions [1923]) and open access databases [24-28] such as the Crystallographic Open Database (COD, that contains currently approximately 85,000 entries $[24,25])$ may be used as sources of these data. For an open access review of crystallographic databases and structural fingerprinting in general, see Ref. [29].

The generated templates are essentially simulations of ED spot patterns in the kinematical limit. The comparison of templates by cross-correlation with experimental ED spot patterns is done on the basis of the spot positions and relative intensities. Other parameters (such as the excitation error) can also be adjusted in order to improve the correlation between the templates and the experimental patterns.
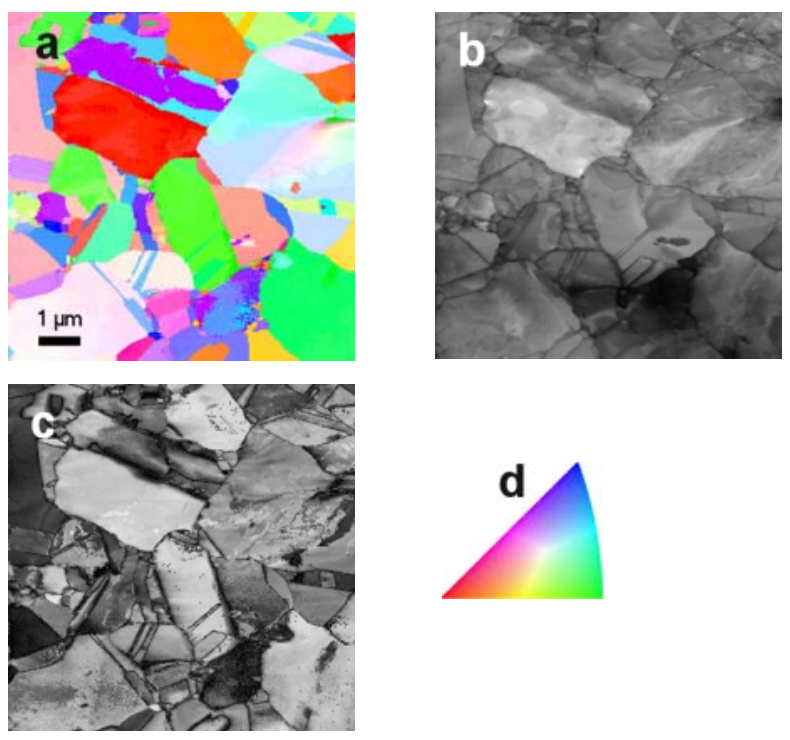

Fig. 3. Orientation map (a) for severely deformed copper showing grain and twin boundaries, (b) correlation index map, (c) reliability map and, (d) color code for the orientation map. 

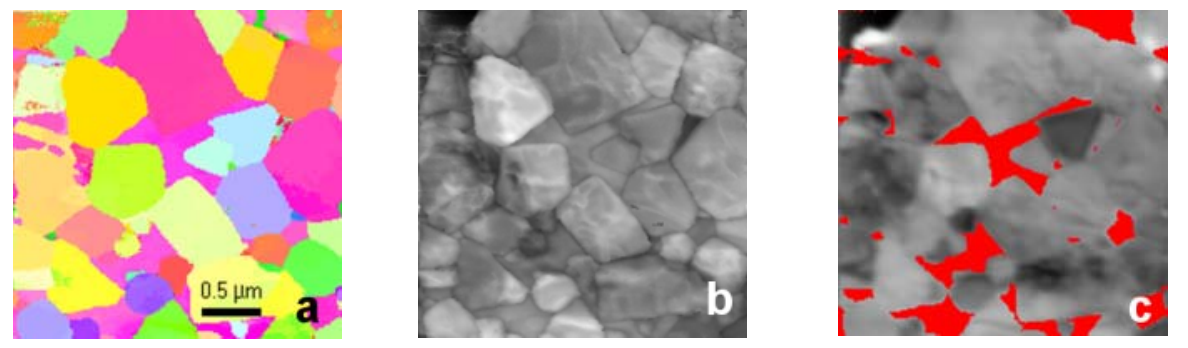

Fig. 4. Orientation map (a) of a sample that contains the two crystal phases (Co and WC), (b) correlation index map and, (c) VBF with Co phase highlighted in red.
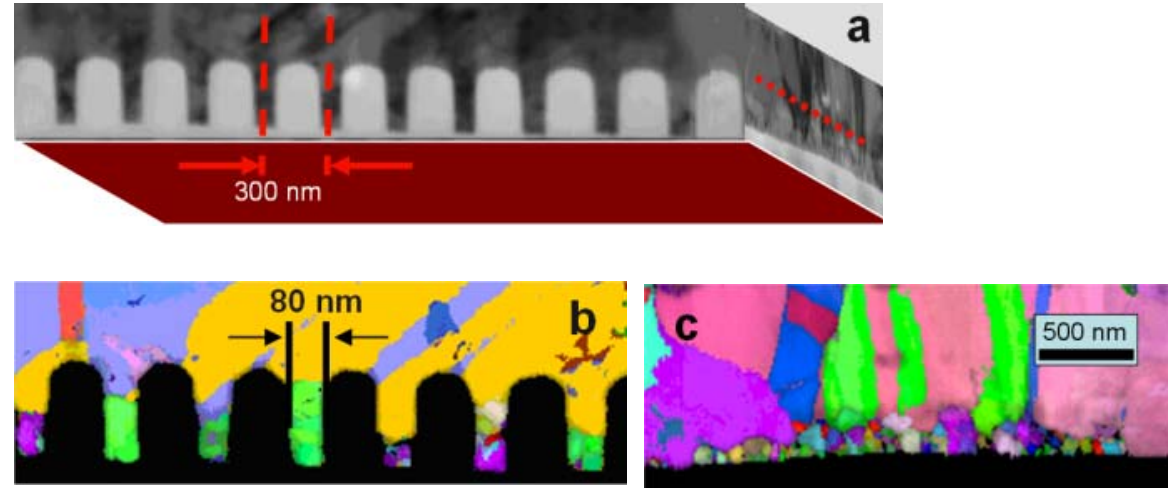

Fig. 5. Orientation mapping for the semiconductor industry: (a) sample overview reconstructed with virtual bright field images of the $80 \mathrm{~nm}$ wide channels filled with copper. (b) and (c) are orientation maps (side and cross views respectively) obtained by scanning the area with a focused beam with a diameter of about $25 \mathrm{~nm}$ in steps of $6.5 \mathrm{~nm}$ and $13 \mathrm{~nm}$, respectively.
In addition to crystal orientation maps (OM), virtual bright field maps (VBF), correlation index maps, and reliability maps can be generated. The VBF map is obtained by plotting the intensity fluctuation of the transmitted beam of the ED spot pattern with the same scanning rate and steps. These maps prove very helpful for the recognition of the scanned area. (Normal parallel illumination bright-field TEM images often show contrast that is difficult to compare with OM maps). The correlation index maps are mainly used to emphasize structural details such as crystals having different orientations. The reliability index (which is roughly analogous to the "EBSD confidence" index) is defined in such a way that it has a minimum when more than one solution is proposed. This can for example happen in the case of two crystals that overlap in the prevalent projection on the screen of the TEM. The highest value of the reliability index is obtained when one assigned solution crystallographic index is much higher that for any other proposed solution. Such reliability maps clearly reveal grain or twin boundaries.

Figures 3 to 5 show typical results for different materials. All experimental ED spot patterns have been acquired with a JEOL JEM 3010 (equipped with a $\mathrm{LaB}_{6}$ gun) at $300 \mathrm{kV}$ and in the nano-probe mode with a nominal probe size of approximately $25 \mathrm{~nm}$. It is important, however, to stress that the dedicated precession/scanning device "DigiSTAR" and the optical CCD camera can be adapted to any older or newer mid-voltage TEM.

\section{Increased reliability for nanocrystal phase and orientation mapping using precession electron diffraction}

Although the orientation and phase mapping coupled with template matching was working satisfactorily without precession for a number of materials, there can be limitations due to the poor quality of experimentally acquired ED spot patterns. This might be the case for thicker crystals where ED patterns may show a combination of a strong diffuse inelastic scattering background, Kikuchi lines, and fainter diffraction spots. Moreover, the reliability of the orientation identification from non-precessed ED spot patterns may be limited by the well known $180^{\circ}$ ambiguity [30].

This ambiguity in the indexing of ED spot patterns arises from the fact that a particular diffraction spot may be indexed as either $(h k l)$ or $(\bar{h} \overline{k l})$ in a plane section through the reciprocal lattice. While one will obtain the same zone axis indices for both sets of indexed reciprocal lattice vectors, an ambiguity will exist as to the azimuthal orientation of the crystals around the zone axis. While this ambiguity is irrelevant for some applications, it becomes important for the determination of grain and phase boundary parameters. Methods to remove this ambiguity are discussed in Ref. [31]. Precession electron diffraction is helpful here because a larger section of the Ewald sphere will be brought in contact with the crystal's reciprocal lattice.

It is well known from that electron precession reduces (non-systematical) dynamical diffraction effects in ED spot patterns significantly, e.g. Ref. [14-16, 32-37], and also suppresses Kikuchi lines and other crystal thickness related effects. Precession ED spot patterns possess an increased number of observable ED spots with respect to standard (stationary or zero-precession-angle) primary electron beam ED spot patterns. Consequently, the results of the template matching technique will be of a higher fidelity when experimentally acquired precession ED spot patterns are used for orientation and phase mapping. In order to achieve this, we used a technique that combines beam scanning and beam precession [13], employing the "DigiSTAR" precession unit.

Dedicated measurements (Fig. 6) reveal that even at small precession angles $\left(\right.$ e.g. $0.2^{\circ}$ to $\left.0.5^{\circ}\right)$, there is a substantial improvement in the matching procedure. The effect is material and orientation sensitive but results typi- 


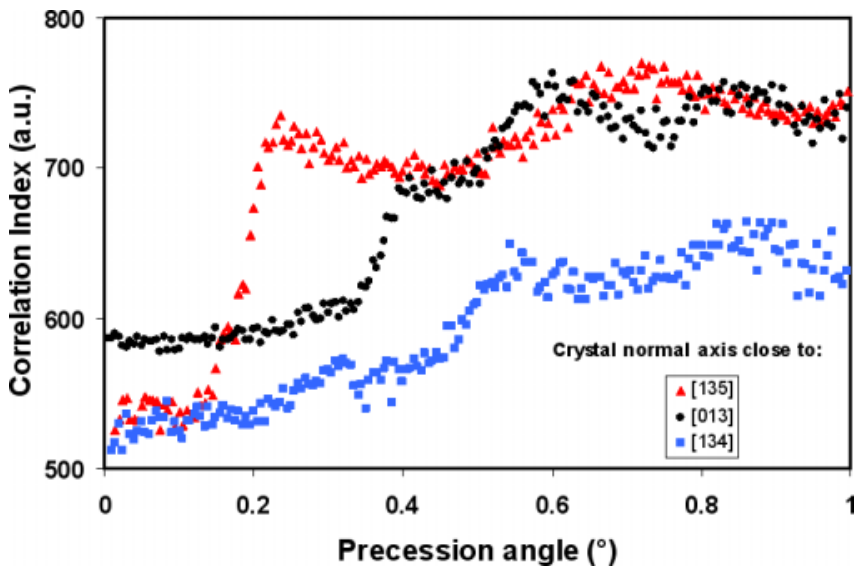

Fig. 6. Correlation index variation with increasing precession angle (from $0^{\circ}$ to $1^{\circ}$ ) for three (randomly selected) copper crystals. A primary electron beam spot size of $25 \mathrm{~nm}$ has been utilized.

cally in an increase of the correlation index from $20 \%$ to $50 \%$. Consequently, crystal orientation and phase indexing will be facilitated when the incident primary electron beam is precessed. Indeed, our previous work has demonstrated an enhanced mapping fidelity due to the application of precession electron diffraction for both nanocrystal phase and orientation maps that have been obtained for steels and severely deformed copper [38] as well as for nanocrystalline iron-oxide minerals [16, 39].

Of scientific interest is here the fact that the trend is observed while the templates were not modified. In turns out that the precessed ED are closer to ideal kinematical conditions that are used to simulate the templates and consequently the improvement is obtained without refinement. A further increases in the map fidelity is expected when the diffraction pattern simulations will include the expected changes in the number and intensity of the diffraction spots as a function of the precession angle. (This is the subject of our ongoing work).
The present work focuses on the capability of precession electron diffraction to overcome the $180^{\circ}$ ambiguity of ED spot patterns. Transformation induced plasticity (TRIP) steels samples containing both ferrite (i.e. a body centered cubic lattice) and austenite (i.e. a face centered cubic lattice) phases are used for this purpose. The precession electron diffraction spot patterns have been recorded with a Philips CM 120 microscope (with a $\mathrm{LaB}_{6}$ gun) that is equipped with the precession diffraction devices "DigiSTAR" [13].

Figures $7 \mathrm{a}$ and $7 \mathrm{~d}$ show experimental ED spot patterns for a ferrite grain recorded without (zero precession angle, Fig. 7a) and with precession of the primary electron beam (Fig. 7d). The beneficial effect of precession electron diffraction to overcome the $180^{\circ}$ ambiguity is clearly revealed by comparing the values of the correlation index as a function of a complete cycle of azimuthal rotation (between the ED spot patterns and their templates) in both case (Fig. 7c and f). While Figure 7c shows two correlation index maxima of essentially the same height that are separated by $180^{\circ}$, there is a significantly higher correlation index for one of these proposed indexing solutions in Fig. 7f. This is obviously due to the precession of the primary electron beam during the recording of this ED pattern. Indeed, a full row of additional spots may be seen at the outer part of a diffraction pattern that is collected with a precessing primary electron beam. This additional row is practically non-existent for a stationary primary electron beam.

Figure 8 shows maps collected on the TRIP steel containing austenite inclusions. An orientation map (Fig. 8a), a correlation index map (Fig. 8b) as well as a crystal phase map superimposed on the reliability map (Fig. 8c) are shown for data that were recorded without precession of the primary electron beam. Apparently, retained austenite phase is detected at the center of the grains, although this crystal phase is expected to exist only between grain
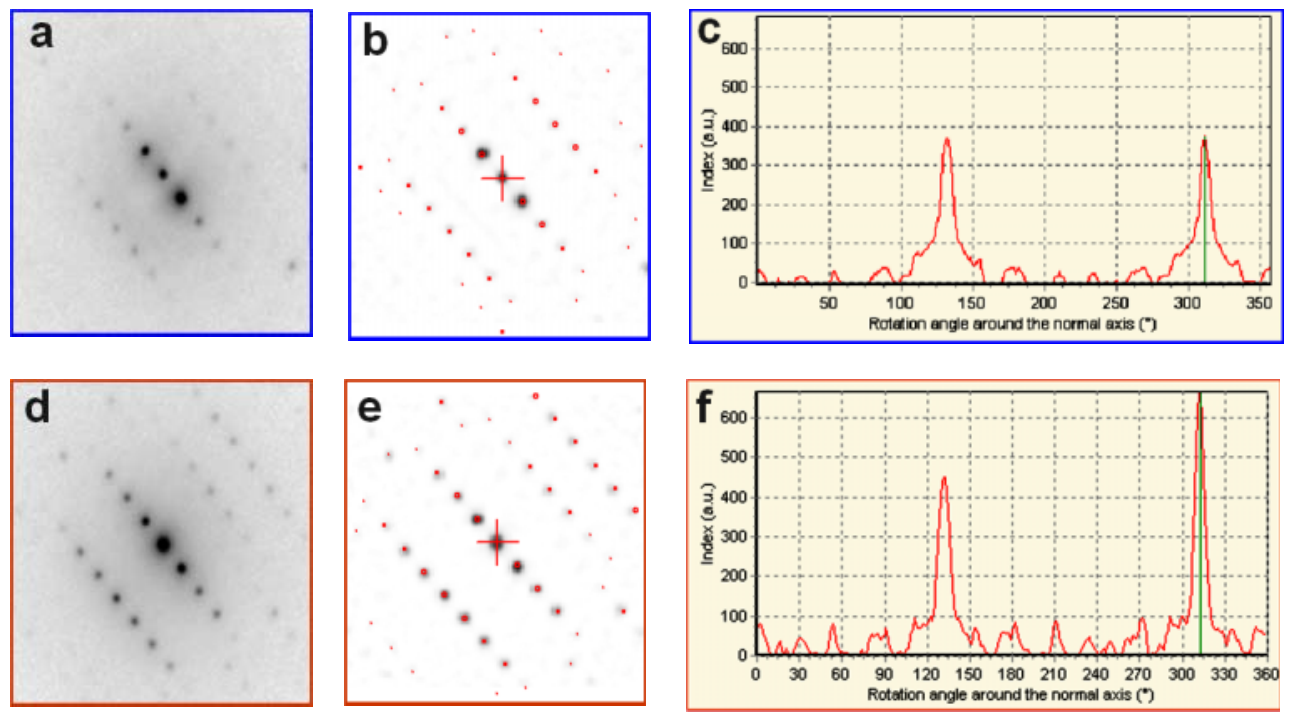

Fig. 7. Tackling the $180^{\circ}$ ambiguity on the example of a ferrite grain in a TRIP steel. Top row: Standard (zero-precession-angle) ED spot pattern (a) and its corresponding matched template (b). The correlation index is nearly 400, but the reliability is close to zero, because two solutions that differ azimuthally by $180^{\circ}$ are equally probable (c), i.e. posses essentially the same correlation index. Bottom row: When a precession ED spot pattern is acquired from the same grain (d), the matching (e) raises the correlation index to about 650 (the precession angle is $1.5^{\circ}$ ), with a reliability index of 28 (f). This means that one of the two indexing solutions is much more likely as shown by the well resolved peak in the azimuthal dependence of the correlation index on the mis-orientation between the precession diffraction pattern and its template. 

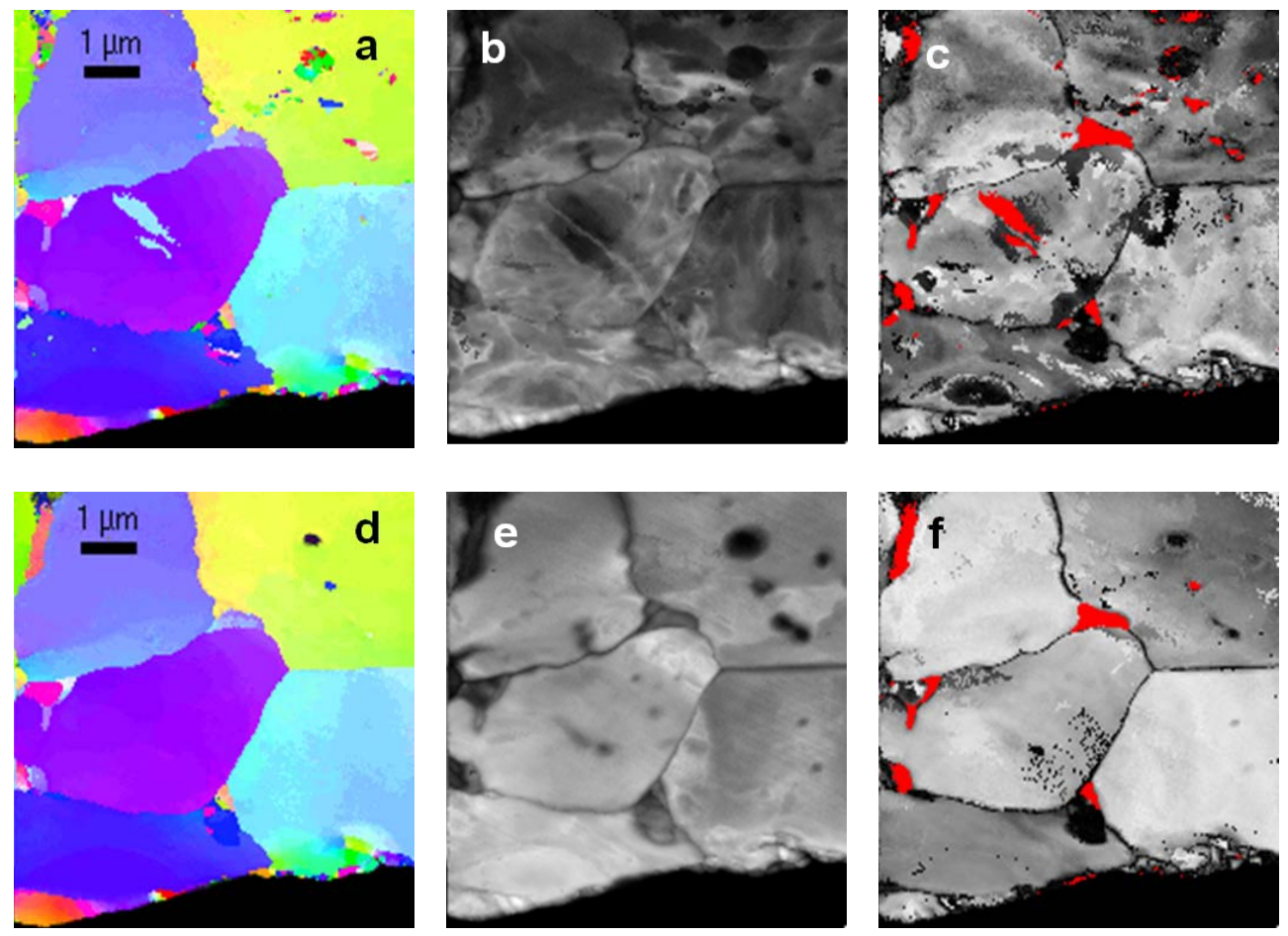

Fig. 8. TRIP ferritic steel containing retained austenite. Top row: results without precession, (a) orientation map, (b) correlation index map, and (c) reliability map with austenite highlighted in red. Bottom row: results with precession (d) orientation map, (e) correlation index map, and (f) reliability map with austenite at the expected positions (highlighted in red). boundaries. Figures $8 \mathrm{~d}$ to $8 \mathrm{f}$ are based on data that were recorded on the same area but with a precessing primary electron beam. With precession, the retained austenite phase is adequately located at the grain boundaries only.

The observation of austenite in ferrite grains in maps that were based on data acquired without precession are, thus, revealed to be errors caused by the limited quality of zero-precession-angle ED spot patterns. Additional studies have shown a systematic positive effect of precession on the fidelity of our results.

\section{Summary and conclusions}

A novel automated crystal phase and orientation mapping system that benefits from the high spatial resolution of transmission electron microscopy has been demonstrated on several examples. This ED spot pattern based system benefits significantly from the precession movement of the primary electron beam around the optical axis of the microscope (and proper de-scanning below the sample) as obtainable with NanoMEGAS's add-on devices to newer and older mid-voltage TEMs. The TEMs to be retrofitted do not need to possess their own dedicated scanning unit.

Acknowledgments. Figures 4 and 5 are extracted from the work of Drs. S. Lay and S. Branstetter from the SIMaP laboratory, Grenoble INP, and their contributions are gratefully acknowledged. The samples for Fig. 3 were supplied by Prof. N. Llorca of the University of Barcelona and her collaboration on this project is acknowledged. The work at Portland State University was supported by grants from the Oregon Nanoscience and Microtechnologies Institute. Additional support from Portland State University's Venture Development Fund is also acknowledged.

\section{References}

[1] Dingley, D.: Progressive steps in the development of electron backscatter diffraction and orientation imaging microscopy. J. Microscopy 213 (2004) 214-224.
[2] Schwarzer, R. A.; Sukkau, J.: Automated crystal orientation mapping (ACOM) with a computer-controlled TEM by interpreting transmission Kikuchi patterns. Mater. Sci. Forum 273-275 (1998) 215-222.

[3] Zaefferer, S.: New developments of computer-aided crystallographic analysis in transmission electron microscopy. J. Appl. Cryst. 33 (2000) 10-25.

[4] Wright, S. I.; Dingley, D. J.: Orientation imaging in the transmission electron microscope. Mater. Sci. Forum 273-275 (1998) 209-214.

[5] Dingley, D. J.; Nowell, M. M.: The use of electron backscatter diffraction for the investigation of nano crystalline materials and the move towards orientation imaging in the TEM. Michrochim. Acta 147 (2004) 157-165.

[6] Dingley, D. J.: Orientation Imaging Microscopy for the Transmission Electron Microscope. Microchim. Acta 155 (2006) 1922.

[7] Li, C.; Williams, D. B.: A new TEM method of interfacial thinfilm characterization. Journal of Electron Microscopy 54 (2005) $57-60$.

[8] Wu, G.; Zaefferer, S.; Advances in TEM orientation microscopy by combination of dark-field conical scanning and improved image matching. Ultramiscroscopy 109 (2009) 1317-1325.

[9] Rauch, E. F.; Dupuy, L.: Rapid spot diffraction pattern identification through template matching. Arch. Metall. Mater 50 (2005) 87-99.

[10] Rauch, E. F.; Duft, A.: Orientation Maps Derived from TEM Diffraction Patterns Collected with an External CCD Camera. Mater. Sci. Forum 495-497 (2005) 197-202.

[11] Gupta V. K., Agnew S. R., Indexation and misorientation analysis of low-quality Laue diffraction patterns. J. Appl. Cryst. 42 (2009) 116-124.

[12] Rauch, E. F.; Veron, M.: Couples microstructural observations of local texture measurements with an automated crystallographic orientation mapping tool attached to a TEM. J. Mater. Sci. Eng. Tech. 36 (2005) 552-556.

[13] www.nanomegas.com, NanoMEGAS-CNRS patent pending technique.

[14] Moeck, P.; Fraundorf, P.: Structural fingerprinting in the transmission electron microscope: overview and opportunities to implement enhanced strategies for nanocrystal identification. Z. Kristallogr. 222 (2007) 634-645; expanded open access version at: arXiv:0706.2021

[15] Moeck, P.; Rouvimov, S.: Structural Fingerprinting of Nanocrystals in the Transmission Electron Microscope: Utilizing Informa- 
tion on Projected Reciprocal Lattice Geometry, 2D symmetry, and Structure Factors, In: Drug Delivery Nanoparticles: Formulation and Characterization (Eds. Y. Pathak, D. Thassu), Informa, 2009 (Drugs and the Pharmaceutical Sciences, Vol. 191), New York, p. 270-313.

[16] Moeck, P.; Rouvimov, S.; Rauch, E. F.; Nicolopoulos, S.: Structural Fingerprinting of Nanocrystals: Advantages of Precession Electron Diffraction, Automated Crystallite Orientation and Phase Maps. In: Electron Crystallography for Materials Research and Quantitative Characterization of Nanostructured Materials (Eds. P. Moeck, S. Hovmoeller, S. Nicolopoulos, S. Rouvimov, V. Petkov, M. Gateshki, P. Fraundorf), Mater. Res. Soc. Symp. Proc. Vol. 1184, Warrendale, PA, 2009), paper 1184-GG03-07.

[17] Hall, S. R.; Allen, F. H.; Brown, I. D.: The Crystallographic Information File (CIF): a new standard archive file for crystallography. Acta Cryst A47 (1991) 655-685.

[18] Hall, S.; McMahon, B.: International Tables for Crystallography, (2005), Volume G, Springer, (ISBN 978-1-4020-3138-0).

[19] http://www.icdd.com.

[20] http://www.nist.gov/srd/nist3.htm.

[21] http://www.fiz-karlsruhe.de/icsd.html; about 3,600 entry on-line demo version freely accessible at: http://icsdweb.fiz-karlsruhe.de/.

[22] http://www.nist.gov/srd/nist83.htm and http://www.nist.gov/srd/ nist84.htm; also free download of an about 3,200 inorganics entry demo version.

[23] http://www.crystalimpact.com/pcd/Default.htm; about 2,600 entry demo version for free download at: http://www.crystalimpact.com/pcd/download.htm.

[24] Gražulis, S.; Chateigner, D.; Downs, R. T.; Yokochi, A. F. T.; Quirós, M.; Lutterotti, L.; Manakova, E.; Butkus, J.; Moeck, P.; Le Bail, A.: Crystallography Open Database - an open-access collection of crystal structures. J. Appl. Cryst. 42 (2009) 726729; open access: http://journals.iucr.org/j/issues/2009/04/00/ kk5039/kk5039.pdf.

[25] http://www.crystallography.net mirrored at: http://cod.ibt.lt (in Lithuania), http://cod.ensicaen.fr/ (in France), http://nanocrystallography.org and http://nanocrystallography.net (both in Oregon, USA), also accessible under a different search surface at: http:// fireball.phys.wvu.edu/cod/ (in West Virginia, USA).

[26] http://nanocrystallography.research.pdx.edu/CIF-searchable/cod.php, data on some 20,000 mainly inorganic crystals with interactive $3 \mathrm{D}$ displays of atomic structures and crystal morphologies.
[27] http://rruff.geo.arizona.edu/AMS/amcsd.php, data on some 10,000 minerals.

[28] http://crystdb.nims.go.jp, data on some 30,000 metals and alloys.

[29] http://en.wikipedia.org/wiki/Crystallographic_database.

[30] Champness, P. E.: Electron Diffraction in the Transmission Electron Microscope. BIOS Scientific Publishers Ltd, Oxford, 2001 (Royal Microscopical Society MICROSCOPY HANDBOOKS, Vo. 47), p. 35-36.

[31] Rauch E. F.; Dupuy L.: Comments on 'On the reliability of fully automatic indexing of electron diffraction patterns obtained in a transmission electron microscope' by Morawiec \& Bouzy (2006). J. Appl. Cryst. 39 (2006) 104-105.

[32] Vincent, R.; Midgley, P.: Double conical beam-rocking system for measurement of integrated electron diffraction intensities. U1tramicroscopy 53 (1994) 271-282.

[33] Gjønnes, J.; Hansen, V.; Kverneland, A.: The Precession Technique in Electron Diffraction and its Application to Structure Determination of Nano-Size Precipitates in Alloys. Microsc. Microanal. 10 (2004) 16-20.

[34] Oleynikov, P.; Hovmöller, S.; Zou, X. D.: Precession electron diffraction: Observed and calculated intensities. Ultramicroscopy 107 (2007) 523-533.

[35] Own, C. S.; Sinkler, W.; Marks, L. D.: Prospects for aberration corrected electron precession. Ultramicroscopy 107 (2007) 534542.

[36] Morniroli, J. P.; Redjaïmia, A.: Electron precession microdiffraction as a useful tool for the identification of the space group. J. Microsc. 227 (2007) 157-171.

[37] Ciston, J.; Deng, B.; Marks, L. D.; Own, C. S.; Sinkler, W.: A quantitative analysis of the cone-angle dependence in precession electron diffraction. Ultramicroscopy 108 (2008) 514-522.

[38] Rauch, E. F.; Veron, M.; Portillo, J.; Bultreys, D.; Maniette, Y.; Nicolopoulos, S.: Automatic Crystal Orientation and Phase Mapping in TEM by Precession Diffraction. Microscopy and Analysis, Issue 93, November 2008, S5-S8.

[39] Rouvimov, S.; Rauch, E. F.; Moeck, P.; Nicolopoulos, S.: Automated Crystal Orientation and Phase Mapping of Iron Oxide Nano-Crystals in a Transmission Electron Microscope. Proc. NSTI 2009, Houston, May 3-7, Vol. I (2009) 421-424, (ISBN: 978-1-4398-1782-7). 